\title{
Reinterpreting recent thymic emigrant function: defective or adaptive?
}

Cody A. Cunningham ${ }^{\mathrm{a}}$, Eric Y. Helm, ${ }^{\mathrm{a}}$, and Pamela J. Fink ${ }^{\mathrm{a}}$

a Department of Immunology, University of Washington, Seattle WA 98109

${ }^{1}$ Present address: Division of Pulmonary Medicine, University of Florida, Gainesville,

FL 32610

Corresponding author: Pamela J. Fink

pfink@uw.edu (email)

206-685-3608 (phone)

206-221-5433 (fax)

Department of Immunology

University of Washington

750 Republican Street

E471 (SLU3.1)

Seattle, WA 98109

C 2017. This manuscript version is made available under the Elsevier user license http://www.elsevier.com/open-access/userlicense/1.0/ 


\begin{abstract}
Recent thymic emigrants (RTEs) are those peripheral T cells that have most recently completed thymic development and egress. Over the past decade, significant advances have been made in understanding the cell-extrinsic and -intrinsic requirements for RTE maturation to mature naïve (MN) T cells and in detailing the functional differences that characterize these two T cell populations. Much of this work has suggested that RTEs are hypo-functional versions of more mature T cells. However, recent evidence has indicated that rather than being defective T cells, RTEs are exquisitely adapted to their cellular niche. In this review, we argue that RTEs are not flawed mature T cells but are adapted to fill an underpopulated $\mathrm{T}$ cell compartment, while maintaining self tolerance and possessing the capacity to mount robust immune responses.
\end{abstract}




\section{Introduction}

T cell development occurs in the thymus via a number of discrete steps to generate $\mathrm{CD}^{+}$and $\mathrm{CD} 8^{+} \mathrm{T}$ cells that are self-tolerant yet capable of recognizing foreign peptide antigen in the context of self-MHC molecules (reviewed in 1). For many years, T cell maturation was thought to be complete once T cells egress from the thymus and populate the secondary lymphoid organs (2). This notion changed in 2004 with the generation of the Rag2p-GFP transgenic (Tg) mouse (3), which allows for the unambiguous identification and retrieval of live RTEs from non-manipulated mice (4). This model does not require surgical intervention (as does intrathymic FITC injection) or PCR-mediated identification (as does TREC-analysis), which had previously limited the types of experiments that could be performed on RTEs (5). Using this model, RTEs were found to have a surface marker phenotype distinct from that of their more mature T cell counterparts. Compared to mature T cells, RTEs are T cell receptor $(T C R)^{\text {hi }}$, CD28 low IL-7R $\alpha^{\text {low }}$, Qa2 ${ }^{\text {low }}$, and CD24hi $(4,6)$. RTEs have also been identified and characterized in humans (see Box 1 and references 711 for the similarities and differences between RTEs in mouse and man). The identification of RTEs as a peripheral T cell subset with a unique surface marker profile raised profound questions about the factors that trigger RTE maturation.

\section{Requirements for the RTE-to-MN transition}

Despite extensive search, the triggers of and requirements for RTE-to-MN maturation have remained frustratingly elusive. RTEs mature to become MN T cells progressively over the course of 2 - 3 weeks, largely as a bulk population, rather 
than enduring selective outgrowth and survival of a small subset of already phenotypically mature RTEs (12). Significant work has shown that the signals known to drive intrathymic maturation and T cell homeostasis do not trigger RTE maturation. The list of molecules that are not required for RTE-to-MN maturation includes self-MHC molecules, the costimulatory molecules CD80 and CD86, and the cytokines and chemokines IL-7, CCL19, and CCL21 $(13,14)$. On the positive side, exit from the thymus, access to the secondary lymphoid organs, and an intact dendritic cell compartment are all required for RTE maturation (12). Recent work has shown that the lipid mediator sphingosine 1-phosphate (S1P), a chemoattractant that helps regulate $\mathrm{T}$ cell trafficking, also regulates mitochondrial metabolism and cell survival (15). Knowing that RTE maturation requires their exit from the blood into the secondary lymphoid organs (thereby moving down an S1P gradient) raises the possibility that changes in mitochondrial metabolism may impact RTE maturation. The unexpected connection between metabolism and RTE maturation is underscored by recent work (16), described more fully below.

The survival of RTEs is clearly required for their maturation and appears to be carefully regulated. On a molecular level, the zinc finger protein Zpf335 (17),

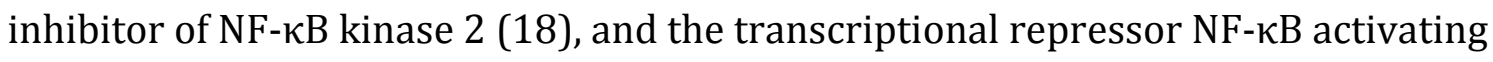
protein or NKAP (19) have been shown to be required for RTE survival during maturation. CD4-cre NKAP cKO mice have a severe defect in the number of naïve peripheral T cells, and NKAP-deficient RTEs are both phenotypically and functionally immature. In the absence of NKAP (20) or the NKAP-associated protein histone deacetylase 3 (21), RTEs have increased surface deposition of natural IgM 
and the complement components $\mathrm{C} 3, \mathrm{C} 4$ and $\mathrm{C} 1 \mathrm{q}$, indicating that RTEs are likely targeted for elimination by the classical complement pathway. Taken together, these data indicate that the ability to inhibit complement deposition is crucial to the survival of newly egressed T cells, and given that survival and maturation are intertwined, factors that regulate elimination by complement also impact RTE maturation (Figure 1).

\section{RTEs display diminished mature $\mathrm{T}$ cell functions}

CD4 ${ }^{+}$RTEs are characterized by reduced activation-induced upregulation of the high-affinity IL-2 receptor (CD25) and diminished proliferative capacity (4). Compared to mature CD4+ ${ }^{+}$cells, RTEs produce less IL-2, IFN- $\gamma$, TNF- $\alpha$, and IL-4 when stimulated under nonpolarizing $\mathrm{T}_{\mathrm{H}} 0$ conditions $(22,23)$. Figure 2 summarizes the different fates that RTEs and mature T cells adopt. Additionally, RTEs produce less IFN- $\gamma$ and IL-17 when stimulated in $\mathrm{T}_{\mathrm{H}} 1$ and $\mathrm{T}_{\mathrm{H}} 17$ polarizing conditions, respectively (23). However, CD4+ RTEs are not universally defective in cytokine production, as RTEs produce more IL-4, IL-5, and IL-13 than mature T cells when stimulated under $\mathrm{T}_{\mathrm{H}} 2$ polarizing conditions. In line with these in vitro results, activated CD4+ RTEs produce more IL-4 and mediate enhanced allergic airway inflammation in vivo (23). Recent work has shown that CD4+ RTEs (defined as the initial wave of $\mathrm{T}$ cells egressing from the thymus of mice post-myeloablation) fail to produce IFN- $\gamma$, IL-10, and TNF- $\alpha$, despite being recruited to the site of granulomatous inflammation during chronic visceral leishmaniasis (24). In spite of this defect in effector cytokine production, $\mathrm{CD} 4^{+} \mathrm{RTEs}$ can still provide protection 
against parasites when transferred into subsequently infected lymphopenic Rag2\%recipients.

CD8 ${ }^{+}$RTEs show similarly diminished effector functions. Under noninflammatory conditions, chicken ovalbumin (OVA)-specific $\mathrm{CD}^{+} \mathrm{T}$ cells produce less IFN- $\gamma$ and IL-2 compared to mature T cells during a primary challenge with ActmOVA splenocytes (25) and in response to pancreatic OVA (26). Even after exposure to a more inflammatory stimulus (Listeria monocytogenes), CD8 ${ }^{+}$RTEs produce less IFN- $\gamma$, TNF- $\alpha$, and IL-2 $(22,25,27)$ on a per cell basis, despite being skewed toward short lived effectors (27). This diminished cytokine profile is maintained into the memory time point (28). However, $\mathrm{CD}^{+} \mathrm{RTEs}$ are not globally defective in terms of effector function, as priming in an inflammatory milieu (in the presence of IL-1 $\beta$, IL-18, and/or IL-33) induces similar CD8+ RTE- and mature T cellgenerated responses (26).

One prevailing interpretation of these compiled data on $\mathrm{CD} 4^{+}$and $\mathrm{CD} 8^{+} \mathrm{RTEs}$ is that RTEs are functionally immature and are simply defective versions of their more mature counterparts. However, recent evidence has prompted us to reassess this interpretation and to foster the emergence of a new paradigm that views RTEs not as defective, but as uniquely attuned to their environment.

\section{RTEs are adapted to maintain immune tolerance}

As newly minted $\mathrm{T}$ cells egress from the thymus and begin to circulate through the secondary lymphoid organs, they encounter a wide range of self- and commensal-derived antigens to which they have not been tolerized. These antigens 
include self-peptides not efficiently presented in the thymus (29), food antigens (30), and antigens derived from commensal microorganisms (31). Failure to become tolerized to these newly encountered antigens is the precipitating event in food allergies and autoimmune diseases such as Type I Diabetes and Crohn's Disease. In line with the idea that newly generated $\mathrm{T}$ cells are exposed to a self antigen-rich environment, it has been shown that PD-1\% RTEs entering a lymphopenic environment cause severe autoimmunity, whereas wild-type RTEs do not (32). Thus, PD-1 expression appears to be critical for dampening this otherwise autoimmune-prone population (Figure 1). The observation that RTEs have longer TCR CDR3 lengths than their more mature T cell counterparts is relevant in this regard (13), given that maturation-associated TCR CDR3 shortening is also correlated with central tolerance (33).

Emerging work has shown that RTEs are exquisitely sensitive to tolerance induced by encounter with extrathymically-expressed antigens. Both $C D 4^{+}$and $C D 8^{+}$ RTEs are hypo-proliferators and poor cytokine producers in the context of a pancreas-expressed self-antigen, rendering them only weakly diabetogenic (26). In an oral tolerance model, CD4+ RTEs (defined as Qa2 ${ }^{\text {low }}$ peripheral T cells) are more likely to become Foxp $3^{+}$regulatory $\mathrm{T}$ cells $\left(\mathrm{T}_{\mathrm{REG}}\right)$ than their more mature $\mathrm{Qa2} 2^{\text {high }}$ counterparts (Figure 2) (34). In the context of lymphopenia, the propensity of CD4+ RTEs to become Foxp $3^{+}$is modulated by the cytokine TGF- $\beta$ (35), that complements their heightened sensitivity to the Foxp3 inducer retinoic acid (34). In addition to their exaggerated tendency toward $\mathrm{T}_{\mathrm{REG}}$ polarization, $\mathrm{CD} 4^{+}$RTEs are also more 
sensitive to $\mathrm{T}_{\mathrm{REG}}$-mediated suppression than are their mature $\mathrm{CD} 4^{+} \mathrm{T}$ cell counterparts (26).

While the mechanistic basis for the tolerance-prone status of RTEs is still the subject of much current research, CD4+ RTEs appear to be uniquely poised for tolerance at the epigenetic level. CD4+ RTEs exhibit hypermethylation of the il-2 promoter relative to mature T cells as well as to their thymic precursors. RTEs appear to be capable of rapidly modulating this promoter methylation state, as they also express high levels of the demethylating enzyme ten-eleven translocation 1 (36). In addition, $\mathrm{CD} 4^{+}$and $\mathrm{CD} 8^{+} \mathrm{RTEs}$ activated to a tissue-restricted model antigen

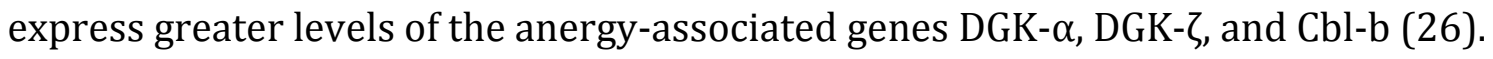
Anergic T cells are known to have reduced rates of aerobic glycolysis (37), a metabolic pathway strictly required for T cell IFN- $\gamma$ production $(38,39)$. With this in mind, we have recently shown that activated CD8 ${ }^{+}$RTEs exhibit reduced rates of aerobic glycolysis compared to their more mature counterparts. Importantly, the rate of aerobic glycolysis (and thus IFN- $\gamma$ production) could be rescued by the addition of exogenous IL-2 (16). These data indicate that metabolism impacts the functional state of RTEs and may directly contribute to their anergy-prone state (Figure 1). Furthermore, as S1P regulates mitochondrial metabolism (and RTEs must exit the S1P rich blood to mature), these data highlight the as yet unexplored idea that changes in cellular metabolism might regulate RTE maturation.

The distinct tissue trafficking preferences of RTEs may also enhance their tolerance-prone status. $\mathrm{CD} 8^{+} \mathrm{RTEs}$ preferentially traffic to the small intestine in a CCR9 and $\alpha_{4} \beta_{7}$ integrin dependent manner (Figure 1) (40), raising the possibility 
that $\mathrm{CD} 8^{+} \mathrm{RTE}$ migration to the gut may facilitate exposure of newly generated CD8 ${ }^{+}$ $\mathrm{T}$ cells to food- and commensal-derived antigens, thereby establishing tolerance (Figure 2). Furthermore, compared to their more mature $\mathrm{T}$ cell counterparts, $\mathrm{CD}^{+}$ RTEs have higher expression of VLA-4 (Figure 1), which enhances their tissue invasiveness and may further expand the range of antigens to which they are exposed (27). A subset of CD4+ RTEs homes to, and is retained in the liver (41), another organ known to establish a tolerogenic environment (42). This population is thought to be prone to autoreactivity, as liver CD4+ RTEs induce more severe autoimmunity when transferred into Rag-/- recipients than do RTEs obtained from secondary lymphoid organs (41). Recent work has shown that stimulated liver RTEs produce IL-10 and exhibit a regulatory cell surface phenotype (43). Additionally, liver-retained RTEs with enhanced FasL and Bim expression undergo higher rates of apoptosis, suggesting that the liver might be a site of their deletional tolerance (Figure 2).

\section{RTEs are well adapted to their distinct cellular niche}

The evidence described above paints a picture of the RTE compartment as comprised of cells that behave as somewhat underperforming mature $\mathrm{T}$ cells yet with some traits that could serve them well in their unique position as newly formed peripheral T cells. Thus, RTEs exhibit diminished proliferation and cytokine production except when encountering antigen in an inflammatory milieu. They skew toward short-lived effector T cells, yet can provide protective immunity during subsequent infections. Activated RTEs have reduced aerobic glycolysis but are 
nevertheless capable of providing immune protection. RTEs are tolerance prone and enjoy enhanced tissue access that may optimize their exposure to extrathymically expressed antigens.

RTEs are also uniquely well-suited to seeding an underpopulated lymphoid periphery, and are incorporated in preference to co-transferred mature T cells, not into a $\mathrm{T}$ cell replete environment, but into an adult lymphoid periphery rendered $\mathrm{T}$ cell lymphopenic by irradiation or genetic ablation (44). CD8 ${ }^{+} \mathrm{T}$ cells in an empty peripheral compartment can undergo lymphopenia-induced proliferation (LIP), resulting in memory conversion and a loss of TCR diversity (45). Converting all entering RTEs from naïve to activated/memory $\mathrm{T}$ cells could prove disastrous in $\mathrm{T}$ cell lymphopenic neonates, but most RTEs entering the periphery of neonatal mice do not undergo LIP. This appears to be a result of IL-7-dependent innate lymphoid cell (ILC) repression of CD8+ RTE LIP, but not proliferation in response to cognate antigen (46). Thus, ILCs play a key role in maintaining the tolerance-prone naïve T cell status of neonatal CD8 ${ }^{+}$RTEs. In line with this, the enhanced engraftment of RTEs relative to mature T cells in lymphopenic hosts is likely a result of augmented RTE survival, rather than increased proliferation (47)

RTEs filling an empty peripheral T cell compartment must also maintain host protection despite relatively low TCR diversity at the cell population level that is generally associated with poor T cell responses due to 'holes' in the TCR repertoire (48). RTEs appear to compensate for this lack of diversity by their enhanced ability to respond to low-affinity ligands, thereby broadening the range of antigens they can recognize (27). These low affinity responses may also play a critical role in 
establishing heterologous immunity to antigens of varying affinity at the memory time point $(49,50)$.

In sum, we propose a reassessment of RTE biology and suggest that, rather than being 'defective' mature T cells, RTEs are uniquely adapted to the environment they encounter upon thymic egress. RTEs efficiently seed a lymphopenic environment without transitioning to activated/memory $\mathrm{T}$ cells. Their trafficking maximizes exposure to extrathymic self- or commensal-derived antigens, and their tolerance-prone status reduces the risk of autoimmunity. This tolerance appears to be enforced by way of an enhanced ability to polarize to a $\mathrm{T}_{\text {reg }}$ phenotype, a greater sensitivity to deletional tolerance, and greater a propensity to become anergic. RTEs are characterized by hypermethylation of cytokine promoters that enforces diminished cytokine production but are poised to reverse this repressed state at the epigenetic level. Furthermore, the dampened pro-inflammatory cytokine production by RTEs is maintained by reduced aerobic glycolysis, yet reversible in the context of an inflammatory milieu. RTEs can provide protective immunity upon reinfection, and are capable of responding to even low affinity antigens, thereby providing comprehensive protection against the pathogenic universe, even in the face of a T cell lymphopenic periphery. RTEs, rather than being mature $\mathrm{T}$ cell "wannabes", are ideally suited for the task at hand, keeping the neonate and lymphoablated adult alive and immunocompetent. 
Box 1: RTEs in mouse and man

$\mathrm{CD}^{+}$RTEs can be identified in humans as CD31+PTK7 ${ }^{+}$peripheral T cells. Recent work has also suggested that human CD4+ RTEs selectively express complement receptor (CR) 1 and CR2. To date, no reliable marker for CD8+ RTEs in humans has been described. On a functional level, mouse and human CD4+ RTEs are similar in that they proliferate less and produce less IFN- $\gamma$, TNF- $\alpha$, and IL-2 than their mature T cell counterparts. Human CD4+ RTEs also produce more IL-8 than mature T cells do. Differences in the methods used to identify and characterize RTEs must be kept in mind when comparing experimental data between mouse and man. Murine RTEs identified by intrathymic FITC injection or from Rag2p-GFP Tg mice have resided in the lymphoid periphery $\sim 24$ hours and $\leq 3$ weeks, respectively. By contrast, human RTEs identified on the basis of PTK7, CR1/CR2, or TRECs have been in the lymphoid periphery for many months. In the neonate, human RTEs enter a less T cell lymphopenic environment than in murine neonates. Some evidence suggests that the adult human naïve T cell compartment may be maintained less by entry of newly generated RTEs and more by homeostatic proliferation of peripheral T cells. 


\section{Figure Legends}

Figure 1 The mechanistic underpinnings of the various RTE and mature T cell fates

Various pathways and molecules influence the fate decisions made by RTEs and MN T cells. The relative expression of molecules known to influence RTE and MN fate decisions is indicated by $(-)$ none, $(+)$ low, and $(++)$ high.

Figure 2 The many fates of antigen-activated RTEs and MN T cells

RTEs and MN T cells can adopt a number of different fates after encountering antigen. These include effector function (and the production of the cytokines IFN- $\gamma$, TNF- $\alpha$, ad IL-2), tolerance in the small intestine, deletion in the liver, anergy, and polarization to the $\mathrm{T}_{\mathrm{REG}}$ lineage. The relative likelihood of RTEs and mature $\mathrm{T}$ cells adopting each fate is correlated with the thickness of the arrow.

\section{Acknowledgements}

This work was supported by National Institutes of Health Grants R01 AI 064318 (to P.J.F.) and T32 DK 007247 (to C.A.C.). The authors would like to thank past and current members of the Fink laboratory. 


\section{References}

\section{** Hsu et al, 2014}

This paper identified NKAP as a critical regulator of RTE survival. This advance identified the first cell-intrinsic factor that specifically regulates RTE survival.

\section{** Friesen et al, 2016}

This paper showed that $\mathrm{CD} 4^{+}$and $\mathrm{CD} 8^{+}$RTEs are prone to anergy in the absence of inflammation. However, in the presence of inflammation, RTEs and mature T cells mount equivalent effector responses.

\section{* Thangavelu et al, 2011}

In line with the idea that RTEs are an autoimmune-prone $\mathrm{T}$ cell subset, this paper shows that PD-1\% RTEs generate profound autoimmune responses.

\section{* Pavia et al, 2013}

This paper shows that $\mathrm{CD} 4^{+}$RTEs are more likely than their mature $\mathrm{T}$ cell counterparts to polarize to the $\mathrm{T}_{\mathrm{REG}}$ subset.

\section{* Cunningham et al, 2017}

This paper shows that activated RTEs have reduced rates of aerobic glycolysis relative to mature $\mathrm{T}$ cells and further highlights the link between altered metabolic state and anergy.

1. Starr, T. K., S. C. Jameson, and K. A. Hogquist. 2003. Positive and negative selection of T cells. Annual Review of Immunology 21: 139-176.

2. Kelly, K. A., and R. Scollay. 1990. Analysis of recent thymic emigrants with subset- and maturity-related markers. International Immunology 2: 419-425.

3. Yu, W., H. Nagaoka, M. Jankovic, Z. Misulovin, H. Suh, A. Rolink, F. Melchers, E. Meffre, and M. C. Nussenzweig. 1999. Continued RAG expression in late stages of B cell development and no apparent re-induction after immunization. Nature 400: 682-687.

4. Boursalian, T. E., J. Golub, D. M. Soper, C. J. Cooper, and P. J. Fink. 2004. Continued maturation of thymic emigrants in the periphery. Nature Immunology 5: 418-425.

5. Fink, P. J., and D. W. Hendricks. 2011. Post-thymic maturation: young T cells assert their individuality. Nature Reviews Immunology 11: 544-548.

6. Hogquist, K. A., Y. Xing, F. C. Hsu, and V. S. Shapiro. 2015. T cell adolescence: Maturation events beyond positive selection. Journal of Immunology 195: 1351-1357.

7. Pekalski, M. L., A. R. Garcia, R. C. Ferreira, D. B. Rainbow, D. J. Smyth, M. Mashar, J. Brady, N. Savinykh, X. C. Dopico, S. Mahmood, S. Duley, H. E. Stevens, N. M. Walker, A. J. Cutler, F. Waldron-Lynch, D. B. Dunger, C. Shannon-Lowe, A. J. Coles, J. L. Jones, C. Wallace, J. A. Todd, and L. S. Wicker. 
2017. Neonatal and adult recent thymic emigrants produce IL-8 and express complement receptors CR1 and CR2. JCI Insight 2.

8. Haines, C. J., T. D. Giffon, L. S. Lu, X. Lu, M. Tessier-Lavigne, D. T. Ross, and D. B. Lewis. 2009. Human $C D 4^{+} T$ cell recent thymic emigrants are identified by protein tyrosine kinase 7 and have reduced immune function. Journal of Experimental Medicine 206: 275-285.

9. Kohler, S., and A. Thiel. 2009. Life after the thymus: CD31+ and CD31- human naive CD4+ T-cell subsets. Blood 113: 769-774.

10. Fink, P. J. 2013. The biology of recent thymic emigrants. Annual Review of Immunology 31: 31-50.

11. den Braber, I., T. Mugwagwa, N. Vrisekoop, L. Westera, R. Mogling, A. Bregje de Boer, N. Willems, E. H. R. Schrijver, G. Spierenburg, K. Gaiser, E. Mul, S. A. Otto, A. F. C. Ruiter, M. T. Ackermans, F. Miedema, J. A. M. Borghans, R. J. de Boer, and K. Tesselaar. 2012. Maintenance of peripheral naive T cells is sustained by thymus output in mice but not humans. Immunity 36: 288-297.

12. Houston, E. G., Jr., R. Nechanitzky, and P. J. Fink. 2008. Cutting Edge: Contact with secondary lymphoid organs drives postthymic T cell maturation. Journal of Immunology 181: 5213-5217.

13. Houston, E. G., Jr., and P. J. Fink. 2009. MHC drives TCR repertoire shaping, but not maturation, in recent thymic emigrants. Journal of Immunology 183: 7244-7249.

14. Houston, E. G. J., T. E. Boursalian, and P. J. Fink. 2012. Homeostatic signals do not drive post-thymic T cell maturation. Cellular Immunology 274: 39-45.

15. Mendoza, A., V. Fang, C. Chen, M. Serasinghe, A. Verma, J. Muller, V. S. Chaluvadi, M. L. Dustin, T. Hla, O. Elemento, J. E. Chipuk, and S. R. Schwab. 2017. Lymphatic endothelial S1P promotes mitochondrial function and survival in naive T cells. Nature 546: 158-161.

16. Cunningham, C. A., T. Bergsbaken, and P. J. Fink. 2017. Cutting Edge: Defective aerobic glycolysis defines the distinct effector function in antigenactivated CD8+ recent thymic emigrants. Journal of Immunology 198: 45754580.

17. Han, B. Y., S. Wu, C. S. Foo, R. M. Horton, C. N. Jenne, S. R. Watson, B. Whittle, C. C. Goodnow, and J. G. Cyster. 2014. Zinc finger protein Zfp335 is required for the formation of the naive T cell compartment. eLife 3.

18. Silva, A., G. Cornish, S. C. Ley, and B. Seddon. 2014. NF-kappaB signaling mediates homeostatic maturation of new T cells. Proceedings of the National Academy of Sciences USA 111: 846-855.

19. Hsu, F. C., A. G. Pajerowski, M. Nelson-Holte, R. Sundsbak, and V. S. Shapiro. 2011. NKAP is required for $T$ cell maturation and acquisition of functional competency. Journal of Experimental Medicine 208: 1291-1304.

20. Hsu, F. C., M. J. Shapiro, M. W. Chen, D. C. McWilliams, L. M. Seaburg, S. N. Tangen, and V. S. Shapiro. 2014. Immature recent thymic emigrants are eliminated by complement. Journal of Immunology 193: 6005-6015.

21. Hsu, F. C., P. J. Belmonte, M. M. Constans, M. W. Chen, D. C. McWilliams, S. W. Hiebert, and V.S. Shapiro. 2015. Histone Deacetylase 3 is required for T cell maturation. Journal of Immunology 195: 1578-1590. 
22. Priyadharshini, B., R. M. Welsh, D. L. Greiner, R. M. Gerstein, and M. A. Brehm. 2010. Maturation-dependent licensing of naive T cells for rapid TNF production. PloS One 5: e15038.

23. Hendricks, D. W., and P. J. Fink. 2011. Recent thymic emigrants are biased against the T-helper type 1 and toward the T-helper type 2 effector lineage. Blood 117: 1239-1249.

24. Moore, J. W., L. Beattie, M. Osman, B. M. Owens, N. Brown, J. E. Dalton, A. Maroof, and P. M. Kaye. 2016. CD4+ recent thymic emigrants are recruited into granulomas during Leishmania donovani infection but have limited capacity for cytokine production. PloS One 11: e0163604.

25. Makaroff, L. E., D. W. Hendricks, R. E. Niec, and P. J. Fink. 2009. Postthymic maturation influences the CD8 T cell response to antigen. Proceedings of the National Academy of Sciences of the United States of America 106: 4799-4804.

26. Friesen, T. J., Q. Ji, and P. J. Fink. 2016. Recent thymic emigrants are tolerized in the absence of inflammation. Journal of Experimental Medicine 213: 913920.

27. Berkley, A. M., and P. J. Fink. 2014. Cutting Edge: $\mathrm{CD}^{+}$recent thymic emigrants exhibit increased responses to low-affinity ligands and improved access to peripheral sites of inflammation. Journal of Immunology 193: 32623266.

28. Deets, K. A., A. M. Berkley, T. Bergsbaken, and P. J. Fink. 2016. Cutting Edge: Enhanced clonal burst size corrects an otherwise defective memory response by CD8+ recent thymic emigrants. Journal of Immunology 196: 2450-2455.

29. Malhotra, D., J. L. Linehan, T. Dileepan, Y. J. Lee, W. E. Purtha, J. V. Lu, R. W. Nelson, B. T. Fife, H. T. Orr, M. S. Anderson, K. A. Hogquist, and M. K. Jenkins. 2016. Tolerance is established in polyclonal CD4(+) T cells by distinct mechanisms, according to self-peptide expression patterns. Nature Immunology 17: 187-195.

30. Pabst, O., and A. M. Mowat. 2012. Oral tolerance to food protein. Mucosal Immunology 5: 232-239.

31. Belkaid, Y., N. Bouladoux, and T. W. Hand. 2013. Effector and memory T cell responses to commensal bacteria. Trends in Immunology 34: 299-306.

32. Thangavelu, G., J. C. Parkman, C. L. Ewen, R. R. Uwiera, T. A. Baldwin, and C. C. Anderson. 2011. Programmed death-1 is required for systemic self-tolerance in newly generated T cells during the establishment of immune homeostasis. Journal of Autoimmunity 36: 301-312.

33. Matsutani, T., T. Ohmori, M. Ogata, H. Soga, S. Kasahara, T. Yoshioka, R. Suzuki, and T. Itoh. 2007. Comparison of CDR3 length among thymocyte subpopulations: impacts of MHC and BV segment on the CDR3 shortening. Molecular Immunology 44: 2378-2387.

34. Bhaumik, S., T. Giffon, D. Bolinger, R. Kirkman, D. B. Lewis, C. T. Weaver, and D. A. Randolph. 2013. Retinoic acid hypersensitivity promotes peripheral tolerance in recent thymic emigrants. Journal of Immunology 190: 26032613.

35. Paiva, R. S., A. C. Lino, M. L. Bergman, I. Caramalho, A. E. Sousa, S. Zelenay, and J. Demengeot. 2013. Recent thymic emigrants are the preferential precursors 
of regulatory $\mathrm{T}$ cells differentiated in the periphery. Proceedings of the National Academy of Sciences of the USA 110: 6494-6499.

36. Berkley, A. M., D. W. Hendricks, K. B. Simmons, and P. J. Fink. 2013. Recent thymic emigrants and mature naïve T cells exhibit differential DNA methylation at key cytokine loci. Journal of Immunology 190: 6180-6186.

37. Zheng, Y., G. M. Delgoffe, C. F. Meyer, W. Chan, and J. D. Powell. 2009. Anergic $\mathrm{T}$ cells are metabolically anergic. Journal of Immunology 183: 6095-6101.

38. Chang, C. H., J. D. Curtis, L. B. Maggi, Jr., B. Faubert, A. V. Villarino, D. O'Sullivan, S. C. Huang, G. J. van der Windt, J. Blagih, J. Qiu, J. D. Weber, E. J. Pearce, R. G. Jones, and E. L. Pearce. 2013. Posttranscriptional control of T cell effector function by aerobic glycolysis. Cell 153: 1239-1251.

39. Peng, M., N. Yin, S. Chhangawala, K. Xu, C. S. Leslie, and M. O. Li. 2016. Aerobic glycolysis promotes $\mathrm{T}$ helper 1 cell differentiation through an epigenetic mechanism. Science 354: 481-484.

40. Staton, T. L., A. Habtezion, M. M. Winslow, T. Sato, P. E. Love, and E. C. Butcher. 2006. CD8 ${ }^{+}$recent thymic emigrants home to and efficiently repopulate the small intestine epithelium. Nature Immunology 7: 482-488.

41. Xu, X., S. Zhang, R. Jin, K. Wang, P. Li, L. Lin, J. Dong, J. Hao, Y. Zhang, X. Sun, X. Pang, X. Qian, J. Zhang, H. Wu, Y. Zhang, and Q. Ge. 2015. Retention and tolerance of autoreactive CD4(+) recent thymic emigrants in the liver. Journal of Autoimmunity 56: 87-97.

42. Crispe, I. N. 2016. Hepatocytes as immunological agents. Journal of Immunology 196: 17-21.

43. Xu, X., R. Jin, M. Li, K. Wang, S. Zhang, J. Hao, X. Sun, Y. Zhang, H. Wu, J. Zhang, and Q. Ge. 2016. Liver sinusoidal endothelial cells induce tolerance of autoreactive CD4+ recent thymic emigrants. Science Reports 6: 19861.

44. Houston, E. G., Jr., L. E. Higdon, and P. J. Fink. 2011. Recent thymic emigrants are preferentially incorporated only into the depleted T-cell pool. Proceedings of the National Academy of Sciences USA 108: 5366-5371.

45. Takada, K., and S. C. Jameson. 2009. Naive T cell homeostasis: From awareness of space to a sense of place. Nature Reviews Immunology 9: 823832.

46. Bank, U., K. Deiser, D. Finke, G. J. Hammerling, B. Arnold, and T. Schuler. 2016. Cutting Edge: Innate lymphoid cells suppress homeostatic T cell expansion in neonatal mice. Journal of Immunology 196: 3532-3536.

47. Kim, H. K., A. T. Waickman, E. Castro, F. A. Flomerfelt, N. V. Hawk, V. Kapoor, W. G. Telford, and R. E. Gress. 2016. Distinct IL-7 signaling in recent thymic emigrants versus mature naive T cells controls T-cell homeostasis. European Journal of Immunology 46: 1669-1680.

48. Yager, E. J., M. Ahmed, K. Lanzer, T. D. Randall, D. L. Woodland, and M. A. Blackman. 2008. Age-associated decline in T cell repertoire diversity leads to holes in the repertoire and impaired immunity to influenza virus. Journal of Experimental Medicine 205: 711-723.

49. Krummey, S. M., R. J. Martinez, R. Andargachew, D. Liu, M. Wagener, J. E. Kohlmeier, B. D. Evavold, C. P. Larsen, and M. L. Ford. 2016. Low-affinity 
memory CD8+ T cells mediate robust heterologous immunity. Journal of Immunology 196: 2838-2846.

50. Knudson, K. M., N. P. Goplen, C. A. Cunningham, M. A. Daniels, and E. Teixeiro. 2013. Low-affinity $\mathrm{T}$ cells are programmed to maintain normal primary responses but are impaired in their recall to low-affinity ligands. Cell Reports 4: 554-565. 


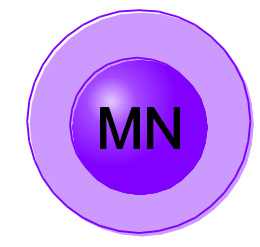

\section{RTE}

+ Exposure
to $\mathrm{Ag}$

\begin{tabular}{|c|c|c|c|}
\hline Glut1 & ++ & + & \multirow{6}{*}{$\begin{array}{l}\text { Metabolic } \\
\text { pathways }\end{array}$} \\
\hline Hexokinase II & ++ & + & \\
\hline CD98 & ++ & + & \\
\hline CD71 & ++ & + & \\
\hline mTORC1 activity & ++ & + & \\
\hline mTORC2 activity & ++ & ++ & \\
\hline pZAP70 & + & ++ & \multirow{2}{*}{$\begin{array}{l}\text { Signaling } \\
\text { molecules }\end{array}$} \\
\hline pERK1/2 & + & ++ & \\
\hline Myc & ++ & + & \multirow{4}{*}{$\begin{array}{l}\text { Transcription } \\
\text { factors }\end{array}$} \\
\hline T-bet & ++ & + & \\
\hline Gata3 & + & ++ & \\
\hline FoxP3 & + & ++ & \\
\hline DGK- $\alpha$ & + & ++ & \multirow{4}{*}{$\begin{array}{l}\text { Anergy } \\
\text { associated } \\
\text { factors }\end{array}$} \\
\hline DGK- $\zeta$ & + & ++ & \\
\hline Cbl-b & + & ++ & \\
\hline PD-1 & - & + & \\
\hline$\alpha_{4} \beta_{7}$ integrin & + & ++ & \multirow{3}{*}{$\begin{array}{l}\text { Trafficking } \\
\text { molecules }\end{array}$} \\
\hline CCR9 & + & ++ & \\
\hline VLA-4 & + & ++ & \\
\hline CD55/DAF & ++ & + & \multirow{2}{*}{$\begin{array}{l}\text { Complement } \\
\text { activity }\end{array}$} \\
\hline CR1/2 & - & + & \\
\hline
\end{tabular}




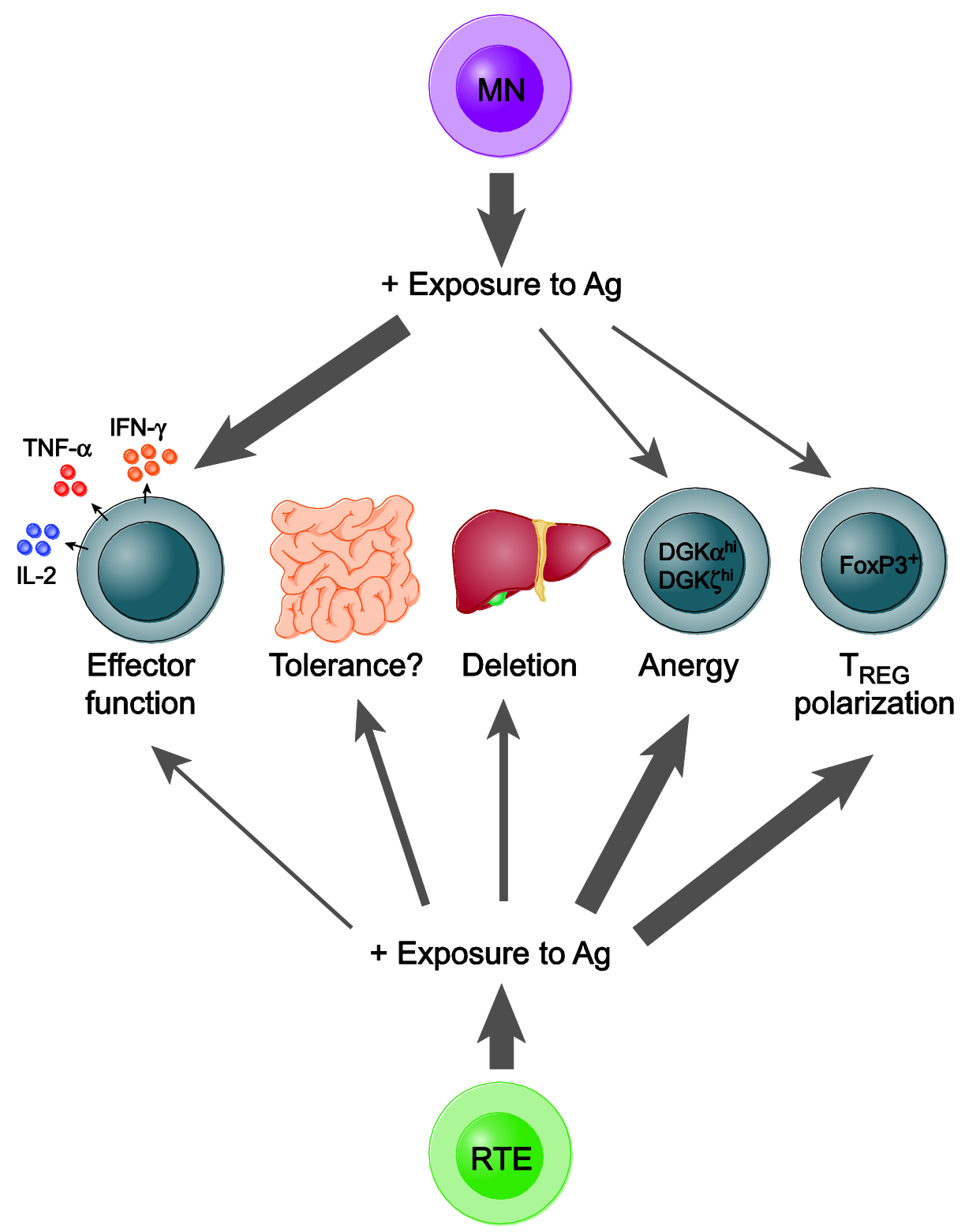

